\title{
AS CARACTERÍSTICAS COMPETITIVAS QUE IMPACTAM A ADOÇÃO DE INOVAÇÃO: UM ESTUDO SOBRE SISTEMAS DE PAGAMENTOS MÓVEIS
}

\author{
Renato Cesar Ottoni Barbosa \\ Doutorando em Administração pela Universidade Presbiteriana Mackenzie - MACKENZIE \\ renatoottoni@yahoo.com (Brasil)
}

\section{Moises Ari Zilber}

Doutor em Administração pela Universidade de São Paulo - FEA/USP

Professor da Universidade Presbiteriana Mackenzie - PPGA/ MACKENZIE

mazilber@mackenzie.com.br (Brasil)

\section{RESUMO}

Este trabalho discutiu as características competitivas que impactam a adoção dos pagamentos móveis pelos clientes, segundo a percepção de executivos que atuam neste setor, objetivando responder à seguinte questão: qual a visão atual dos executivos relacionados ao mercado brasileiro de pagamentos móveis sobre os elementos de adoção da inovação pelo consumidor? O trabalho também objetivou contextualizar e descrever quais são os participantes deste esquema. O estudo, de caráter exploratório, foi desenvolvido por meio de entrevistas estruturadas, com dez executivos representantes dos participantes do mercado de pagamentos móveis. Os dados obtidos foram apresentados pela técnica da matriz importância-desempenho e os resultados indicaram que conveniência, compatibilidade com os hábitos atuais do usuário e a facilidade de uso são as características competitivas das soluções de pagamentos móveis que devem merecer maior atenção dos desenvolvedores de soluções, para aumentar o nível de adoção da inovação pelo consumidor.

Palavras Chave: Administração; Inovação; Adoção de Inovação; Pagamento Móveis; Matriz Importância-desempenho. 


\section{INTRODUÇÃO}

A inovação na indústria dos meios de pagamentos eletrônicos pode ser representada pelo aparecimento de uma nova maneira de realização de transações financeiras, chamada de pagamentos móveis (mobile payments). Dahlberg et al. (2007) destacam que os telefones móveis transformaram a telefonia de maneira profunda, ao oferecerem funcionalidades que superam as necessidades simples de uso do telefone. Diversos serviços de valor agregado para os usuários vêm sendo criados e esta capacidade de realização de transações financeiras faz com que este instrumento entre na cadeia da indústria, concorrendo com os participantes atuais, notadamente cartões de crédito. Dewan e Chen (2005) complementam que atualmente a indústria de pagamentos está experimentando a convergência de tecnologia para processamento de transações. Os autores defendem que existem benefícios reconhecidos para todos os participantes do setor e que diversas mudanças no comportamento dos consumidores parecem favorecer a adoção de novas formas criativas de métodos de pagamento ao redor do mundo.

O segmento do setor de serviços de meios de pagamentos tradicionais é caracterizado pela presença de diversos participantes. Os emissores são as instituições responsáveis pelo relacionamento com os portadores de cartões de crédito, concedendo crédito e possibilitando o uso, com respectiva cobrança da fatura das transações. Os credenciadores são responsáveis pela administração do contrato com o estabelecimento comercial, mantendo uma rede de captura para uso dos portadores. Finalmente, partes importantes do segmento são as bandeiras de cartões, que possuem a marca e definem as regras e o funcionamento do negócio. Novos participantes passam a fazer parte do setor, com a inclusão dos operadores de telefonia móvel, assim como os fornecedores de equipamentos, de software e de serviços ligados a este tipo de operação.

Chen e Adams (2005) comentam que os recentes desenvolvimentos de tecnologias de comunicação móveis e sem fio têm alterado a vida diária das pessoas. O crescimento do uso dos equipamentos móveis, assim como a alta taxa de penetração destes instrumentos na sociedade, têm tido um impacto positivo no desenvolvimento de aplicação de pagamentos móveis. Dahlberg et al. (2007) complementam que os números de telefones móveis utilizados pelas pessoas excedem muito qualquer outro instrumento usado para comercializar, vender, 
produzir e entregar produtos e serviços para os consumidores. Os autores destacam que estes desenvolvimentos representam uma oportunidade lucrativa para provedores dos serviços, assim como para os estabelecimentos comerciais.

Alguns estudos refletem que a popularidade do uso destes novos instrumentos propicia a necessidade de identificar e entender os determinantes da aceitação dos consumidores para os sistemas de pagamentos móveis.

Dahlberg et al. (2003) chamam a atenção para o fato de que até agora os serviços de pagamentos móveis têm falhado em seduzir os consumidores, aparentemente por não atingir as suas necessidades. Sendo assim, os autores destacam que um melhor entendimento das motivações para a adoção desta forma de inovação é necessário, para desenvolver e lançar serviços de pagamentos móveis com sucesso.

Algumas questões são colocadas por Zmijewska et al. (2004b). Porque os usuários não estão usando os novos sistemas? O que fazer para que eles aceitem a nova maneira de pagar? Como podem ganhar a aceitação dos clientes?

Com base nas pesquisas acima citadas, pretendeu-se identificar as principais características competitivas que impactam a adoção pelos usuários.

Dahlberg et al. (2007) destacam que os ambientes culturais e sociais afetam os hábitos de consumo, comportamentos de compra e as necessidades para novos serviços de pagamento, incluindo diferentes maneiras de fazê-lo de acordo com o país analisado. Diferenças culturais entre países em desenvolvimento e desenvolvidos também podem afetar a demanda e aceitação dos serviços de pagamentos móveis.

No Brasil, uma série de iniciativas tem sido oferecida para os consumidores, tanto pelos participantes tradicionais do segmento de meios de pagamentos, quanto pelos novos entrantes. O desafio de aceitação dos produtos e serviços é grande. Pretendeu-se avaliar, portanto, qual a importância de cada característica de adoção pelo usuário, assim como analisar o atual desempenho destas características em relação às demais ofertas de pagamentos móveis considerando-se ainda os meios de pagamentos tradicionais.

Nesse sentido, buscou-se avaliar a percepção de diversos executivos de diferentes participantes da cadeia do mercado brasileiro (tradicionais e entrantes), para atender o objetivo final de discutir a adequação de cada uma das características à oferta ao consumidor então encontrar os itens que necessitem de maior atenção para adequação entre a importância percebida para os clientes finais e o desempenho em relação às outras ofertas.

Revista de Administração e Inovação, São Paulo, v. 10, n.3, p.89-114, jul./set. 2013. 
Sendo assim, o problema delimitado para este estudo - qual a visão competitiva atual dos executivos relacionados ao mercado brasileiro de pagamentos móveis, sobre os elementos de adoção pelo consumidor foi avaliado pela classificação das variáveis identificadas na revisão de literatura, a seguir apresentada.

\section{2}

\section{FUNDAMENTAÇÃO TEÓRICA}

2.1

\section{Inovação e sua adoção}

Segundo a Organisation of Economic Co-Operation and Development [OCDE] (1984), inovação é a implementação tecnológica de novos produtos e processos ou suas melhorias tecnológicas efetivamente implementadas (introduzidas no mercado ou usadas no processo de produção). Afuah (1998) complementa que a inovação é o uso de um novo conhecimento para oferecer um novo produto ou serviço, porém destaca que é necessário que os consumidores queiram o produto. $\mathrm{O}$ autor defende que a inovação está ligada ao conhecimento de marketing, que inclui as expectativas, preferências, necessidades e vontades dos clientes.

A inovação tecnológica pode ser realizada por meio da introdução de um novo produto, que tenha características ou usos diferentes dos produtos anteriormente produzidos (novas tecnologias, recombinação de tecnologias existente ou resultantes do uso de novas tecnologias). A inovação tecnológica pode ser representada também pela melhoria em produtos, realizada em um produto existente com performance melhorada. Finalmente, a inovação pode ser realizada nos processos, com métodos de produção novos ou melhorados (incluindo processo de entrega), ou ainda mudanças em equipamentos ou na organização da produção (OCDE, 1984).

Tidd et al. (2005) destacam que existe uma dificuldade grande para entender como seria a configuração "certa" de necessidades de meios tecnológicos e mercados. Os autores destacam que existem experimentações intensas, que podem gerar muitos fracassos, mas que resultarão em um aprendizado acelerado por parte dos participantes. Estes autores destacam a importância de que os experimentos sejam bem concebidos e controlados para minimizar a incidência de falhas e caso estas ocorram, que as lições sejam aprendidas para evitar novos 
erros futuros.

Um dos grandes desafios de um processo de gerenciamento de inovação é a sua adoção. Frambach e Schillewaert (2002) destacam que adoção é a decisão de qualquer indivíduo em fazer uso de uma inovação, Segundo os autores, é o processo pelo qual um indivíduo primeiro tem conhecimento de uma inovação, para formar uma atitude sobre esta, até tomar uma decisão entre adotar ou rejeitar, para a sua implementação e a confirmação desta decisão.

\section{2}

\section{Adoção de inovação em pagamentos móveis}

Davis (1989) desenvolveu um modelo para a aceitação dos usuários para novas tecnologias, chamado de TAM (Technology Acceptance Model). Este modelo tem sido usado como base para diversos estudos de aceitação de tecnologia, podendo, segundo Chen e Adams (2005) ser uma ferramenta para analisar as intenções de consumidores em relação à aceitação de pagamentos móveis. Zmijewska et al. (2004b) complementam que este modelo de aceitação tem sido usado de forma extensiva nas análises de aceitação de diversos sistemas de informações, e que como já foi testado e verificado em diversos estudos, beneficiarão a análise de pagamentos móveis. Segundo este autor, o modelo é baseado na crença de que a decisão da aceitação ou rejeição é decorrente de diversos fatores individuais, que poderiam ser identificados e medidos.

No modelo inicial, Davis et al. (1986) propõe que a aceitação de tecnologia é direcionada inicialmente por dois fatores que a tecnologia ofereceria ao usuário. O primeiro fator, "Utilidade percebida" refere-se ao grau em que uma pessoa acredita que utilizando um sistema específico seria facilitada sua performance para realização de um certo trabalho. $\mathrm{O}$ outro fator seria "Facilidade de uso percebida" que é o grau que o usuário acredita que a utilização de uma certa aplicação será feita sem esforços.

Estes dois fatores podem afetar em conjunto a atitude de uma pessoa na utilização de um certo sistema, gerando uma intenção de uso do sistema, culminando em sua utilização.

$\mathrm{Na}$ aplicação deste modelo, diversas medidas têm sido usadas. No indicador de utilidade percebida, podem ser utilizadas as métricas de aumento da performance, aumento da produtividade, efetividade, utilidade geral, economia de tempo e melhoria da performance no trabalho. Em relação à facilidade de uso percebida, podem ser avaliadas a facilidade de 
aprendizagem, a facilidade de controle, facilidade de entendimento, facilidade de uso, claridade e flexibilidade (Dahlberg et al., 2003).

Zmijewska et al. (2004b) concluem que a utilização do modelo TAM para atividades do cotidiano diário é apropriada, mas que quando este modelo é usado em novos campos de estudo, é necessário que seja adaptado ou expandido para encontrar as características de um serviço específico. O modelo TAM serve para ser usado como base para a criação de um modelo estendido com os construtos específicos de tecnologias mais novas.

Dahlberg et al. (2007) destacam que quando se analisa a pesquisa de consumidores de pagamentos móveis, percebe-se que a maioria dos estudos investigou fatores de adoção, com a utilização do modelo TAM, com as modificações que adéquem o estudo para este contexto. Os estudos trouxeram à luz, as características dos novos serviços de pagamentos que estimulavam ou inibiam a sua adoção. Os consumidores podem estar dispostos a aceitar algumas características que não atendam totalmente suas necessidades, porém podem rejeitar um serviço por uma fraqueza específica. Os novos indicadores servem, portanto, para serem usados como critérios de avaliação para classificação de sua importância relacionada ao que realmente importa para os consumidores.

\section{3}

\section{Características competitivas que impactam a adoção de pagamentos}

\section{móveis}

Alguns estudos propuseram fatores de adoção adicionais que seriam específicos ao ambiente de pagamentos móveis, como custos, externalidades de rede, confiança e segurança. Segundo a análise de Dahlberg et al. (2007), os fatores de adoção mais importante para estes serviços seriam a facilidade de uso, utilidade, compatibilidade, confiança e segurança.

São destacadas a seguir, as características competitivas que impactam a adoção de pagamentos, segundo a revisão de literatura realizada.

Custos: Os custos de uma transação de pagamento incluem os diretos da transação, assim como os fixos de uso. Adicionalmente, Segundo Pousttchi (2003), devem ser avaliados os custos da infra-estrutura tecnológica para o cliente, custos de equipamentos, como por exemplo, um novo telefone celular. Mallat (2006) destaca que a adoção do consumidor é diretamente afetada pelo custo das transações de pagamento se este é repassado para os 
clientes. Como os clientes que compram em canais eletrônicos são atentos para os preços, o custo das transações de pagamentos móveis deve ser baixo o suficiente para que o total da compra seja competitivo com os preços de no mundo físico. Karnouskos (2004) chama a atenção para o fato de que novos sistemas devem ter um custo efetivo melhor que as soluções legadas, podendo até ser maior, porém gerando um valor adicional, como redução de fraudes e maior segurança para o consumidor, ou ainda, oferecer uma solução mais adequada para os processos do consumidor, justificando assim a sua existência.

Compatibilidade: Segundo Mallat (2006) esta variável relaciona uma inovação e os valores, experiências e necessidades do potencial consumidor. Quando uma inovação proporciona produtos e serviços complementares ou alternativos e um esforço pequeno é necessário para aprender a operar ou para mudar um comportamento, o consumidor potencial tem uma maior probabilidade de aceitação da inovação, complementam Chen e Adams (2005). Para sistemas de pagamentos, a habilidade dos consumidores em integrá-los em sua vida cotidiana é um importante aspecto de compatibilidade, destaca Mallat (2006). A utilização de sistemas de pagamentos móveis poderá requerer somente um entendimento dos procedimentos operacionais, e não alterar o comportamento e hábitos dos usuários nas atividades inerentes a eles. Neste caso, esta compatibilidade deverá ter um efeito positivo sobre a intenção dos usuários sobre o novo sistema. A compatibilidade do pagamento móvel com os hábitos atuais do usuário influenciará a adoção pelos usuários.

Confiança: Karnouskos (2004) destaca que ao se cadastrarem em um sistema de pagamentos móveis, os usuários esperam confiar totalmente no sistema. Permitir acesso `a sua conta-corrente ou a sua conta de cartão de crédito para uma empresa de software, por exemplo, não é a mesma coisa, na cabeça da maioria dos usuários, do que dar o mesmo acesso a uma entidade estabelecida como de sua confiança caso dos bancos. Sendo assim, somente se as bases do sistema de pagamento eletrônico forem comparáveis com práticas bancárias testadas e verdadeiramente seguras, sera possível aos usuários adotarem esta solução. Portanto, todos os passos do processo devem ser seguros e confiáveis tanto em uma perspectiva tecnológica quanto social. Dahlberg et al. (2003) complementam que a confiança percebida indica se a pessoa avalia uma tecnologia específica como segura e confiável. Alguns pontos destacados por estes autores decorrentes de suas pesquisas, seriam a falta de registros de transações e de documentação, o que dificultaria conferir os pagamentos e ter uma prova de sua efetivação. Adicionalmente, a imprecisão das transações, quando o portador Revista de Administração e Inovação, São Paulo, v. 10, n.3, p.89-114, jul./set. 2013. 
não tem a confirmação se o pagamento aconteceu ou não, configura-se como mais um ponto de preocupação. Zmijewska et al. (2004b) chamam a atenção para o fato de que, como em qualquer sistema de pagamento, confiança tem uma alta importância para os pagamentos móveis. Confidencialidade dos dados, confirmação de pagamentos e possibilidade de cancelamento são considerados importantes por usuários entrevistados. Os autores concluem que a confiança é influenciada pela anonimidade, segurança, disponibilidade, quantidade de controle que o usuário tem, assim como a reputação que a entidade que oferece o sistema tem.

Conveniência: $O$ critério desta categoria tem três domínios, segundo Pousttchi (2003): a conveniência da operação por si só, a fase de iniciação antes do primeiro uso e finalmente a cobertura física do sistema com amplitude geográfica que garantirão locais para utilização. O autor defende que depois de problemas de segurança e custo, este seria o ponto mais importante de atenção. A conveniência da operação pode ser representada pelo manuseio facilitado e processamento rápido, a questão inicial pela não necessidade de pré-registro ou de instalação de softwares, enquanto a cobertura é representada pelo alto número de lojas que aceitam o produto ou a utilização internacional da solução. Karnouskos (2004) complementa que a conveniência determinará se os consumidores começariam a usar os serviços, representada pela quantidade de produtos e serviços que poderiam ser comprados e a disponibilidade geográfica do serviço. Mallat (2006) exemplifica o aumento de conveniência para o usuário de pagamentos móveis, pela redução da necessidade de moedas e dinheiro em transações de pequeno valor e um aumento das possibilidades disponíveis de pagamentos. Segundo Dewan e Chen (2005), o consumidor não gosta de esperar ou gastar tempo em tarefas que não percebe como necessárias e redundantes. Os usuários prefeririam transações tranqüilas e automáticas, a ficar com a frustração de ter que esperar por troco, ou precisar ter o valor exato a pagar. Adicionalmente, seria eliminada a necessidade de ir até o caixa da loja, ter que esperar o cartão ser passado e finalmente assinar o recibo para autenticação da transação.

Externalidade de rede e criação de massa crítica: Mallat (2006) explica que todos os sistemas de pagamentos demonstram externalidades de rede, uma vez que o valor para um usuário individual aumenta à medida que mais usuários começam a utilizar o sistema. $\mathrm{O}$ autor continua explicando que a decisão do consumidor para adotar um certo sistema de pagamentos é significantemente afetada pela quantidade de outros consumidores e 
estabelecimentos comerciais que estejam usando. Um dos motivos que determinariam a descontinuidade de diversos sistemas de pagamentos seria a falha na construção da massa critica. O sistema de pagamento móvel configura-se como um novo modelo que se inicia no mercado sendo, portanto um fator crítico do sucesso atingir-se uma base inicial grande o suficiente de consumidores e lojas adotando a solução.

Facilidade de uso: Os estudos iniciais de adoção utilizando a metodologia TAM já provaram um relacionamento entre a facilidade de uso e a aceitação do usuário, em diversos campos de pesquisa. Estudos sobre pagamentos móveis também sugeriram que este construto caracteriza-se como um importante fator de sucesso. Sendo assim, Zmijewska et al. (2004b) defendem que quando o consumidor perceber que um modelo de pagamento móvel for fácil de usar, ele estará mais disposto a utilizá-lo. Por outro lado, Chen e Adams (2005) comentam que quando o desenho de uma inovação for sofisticado e complicado, os adotantes potenciais precisarão de mais conhecimento e instruções para operar ou entender os produtos e serviços, diminuindo desta forma, a taxa de adesão a inovação. A simplicidade da solução determinará de maneira importante quem utilizará o sistema, destaca Karnouskos (2004). A interface deverá ser amigável e deve evitar limitações das características dos aparelhos como, por exemplo, telas e teclados pequenos, complementa Mallat (2006). Karnouskos (2004) chama a atenção para a necessidade de que a curva de aprendizagem se situe perto de zero, para aumentar a facilidade de uso e conveniência do usuário. Adicionalmente, o usuário deveria ter uma capacidade alta de personalizar a aplicação e o serviço, para integrar-se rapidamente com suas atividades de pagamentos diários. Chen e Adams (2005) concluem que os serviços de pagamentos móveis não devem ser inovações complexas, objetivando prover um serviço alternativo e conveniente para os consumidores. Sendo assim, os provedores de soluções devem considerar a facilidade de uso e oferecer uma solução simples.

Mobilidade: Mobilidade é uma categoria específica dos serviços móveis, sendo também uma característica única quando comparada com as de outros meios de pagamentos, destacam Zmijewska et al. (2004b). O grande benefício deste construto é a possibilidade de utilização em qualquer lugar. Este fator não será satisfeito se não houver uma área de cobertura suficiente ou não existir operadoras suficientes oferecendo o serviço, existindo uma pressão pelos usuários por soluções de roaming ou cooperativas com outras operadoras. Estas soluções de roaming incluiriam tanto a vontade do usuário em fazer pagamentos enquanto estiver viajando fora de sua rede de cobertura ou poder faze-lo em outras redes. Um outro Revista de Administração e Inovação, São Paulo, v. 10, n.3, p.89-114, jul./set. 2013. 
ponto crucial destacado por Mallat (2006), é o fato do aparelho ter a vida útil de sua bateria pequena, ou descarregar muito rápido. Um dos pontos de preocupação de usuários, identificado na pesquisa de Dahlberg et al. (2003), seria a confiança nos aparelhos e na rede móvel, de certa forma consideradas não confiáveis para pagamentos, uma vez que a bateria do aparelho pode descarregar ou a conexão de rede pode falhar no meio de uma transação.

Observação de utilização: Chen e Adams (2005) afirmam que sendo os pagamentos móveis uma inovação relativamente recente para a maioria dos consumidores, provavelmente se a capacidade da utilização desta inovação puder ser facilmente mostrada para outros, a adoção do sistema de será aumentada. Os autores continuam defendendo que uma inovação deve atrair a atenção de um grupo de usuários específicos, para que eles tenham o conhecimento do serviço. Segundo os autores, pagamentos móveis seriam bem vistos quando um usuário utilizando o serviço em público, propicia a sua divulgação uma vez que os potenciais usuários podem observar facilmente a inovação.

Privacidade: Karnouskos (2004) destaca que a maioria dos programas de pagamentos móveis avaliados por ele, requer aos usuários que se registrem, informando dados privados, porém limitam o controle que o usuário tem em relação a estes dados. A tendência atual é de que as informações não sejam armazenadas de forma centralizada, sendo distribuídas pelo mundo afora. Adicionalmente, o número de pontos de acessos a estes dados vem aumentando, uma vez que têm que ser compartilhados com as partes envolvidas. Entretanto, prossegue o autor, privacidade é crucial para o sucesso futuro dos pagamentos móveis, no sentido de substituir o dinheiro vivo atual. O autor conclui que privacidade e anonimidade são fundamentais para garantir a confiança dos clientes, estabelecendo uma base em que possam aparecer novos serviços de valor agregado que utilizem dados sensíveis pessoais. Mallat (2006) destaca que o comprometimento de privacidade é percebido como um risco por alguns usuários pesquisados, que conseqüentemente não estariam dispostos a compartilhar suas informações com o provedor da solução. A preocupação caso algumas transações possam ser rastreadas, com um mau uso de suas informações pessoais, ou que os usuários possam começar a receber uma quantidade enorme de propagandas, no caso de se registrarem no sistema, devem diminuir a aceitação do sistema de pagamento por parte do usuário (Dahlberg et al., 2003). Sendo assim, as soluções de pagamentos móveis devem proporcionar um controle de privacidade específico para cada usuário, de modo a possibilitar pagamentos 
anônimos (como é feito com o dinheiro), destacam Dewan e Chen (2005). Zmijewska et al. (2004b) concluem que a confiança em qualquer sistema de pagamento é influenciada pela anonimidade, confidencialidade, integridade de dados e a quantidade de controle que o usuário tem.

Qualidade do sistema: Dois fatores podem ser considerados como indicadores de qualidade de sistema, na visão dos usuários, segundo Lee et al. (2004). O primeiro fator seriam as infraestruturas tecnológicas da rede, que removeriam as barreiras para o uso. As capacidades dos aparelhos também determinariam o tipo e freqüência de uso de pagamentos móveis. Adicionalmente, como o sistema opera em redes abertas, as capacidades de segurança também influem no uso destas soluções. Os autores destacam que países com infra-estruturas de telefonia sem fio muito bem desenvolvidas, como Japão e Coréia do Sul, têm demonstrado um rápido crescimento dos negócios em pagamentos móveis. Outro ponto importante destacado pelos autores é a qualidade das aplicações que possibilitam as transações de pagamentos móveis, disponibilizando ferramentas para os usuários. As aplicações devem possibilitar ao usuário gastar, guardar e transportar o seu dinheiro. Devem também demonstrar a finalização destes pagamentos, provendo formas de registro das mesmas. Todas as soluções devem ser ininterruptas, operando 24 horas por dia, sete dias por semana. Os autores concluem que, quando os sistemas oferecem estas capacidades, aumenta a confiança no uso de pagamentos móveis, fazendo com que os usuários tenham um maior grau de adoção.

Rapidez da transação: Dewan e Chen (2005) destacam que um dos pontos que podem trazer uma maior adoção do sistema de pagamentos móveis, é a percepção da medida em que estes pagamentos vão aumentar a velocidade das transações. Karnouskos (2004) complementa que os novos meios de pagamento deverão diminuir o tempo de transação e automatiza-la, mantendo os níveis de segurança requeridos. Como comparação, Zmijewska et al. (2004b) explicam que o tempo médio de duração de uma transação de cartão de crédito é de menos de trinta segundos, enquanto em dinheiro toma menos de dez segundos. Os autores concluem que parece ser importante comparar os novos sistemas de meios de pagamentos com as transações de cartões de crédito e débito, caso estes se tornem, novos meios de pagamento.

Segurança: Heijden (2002) destaca que segurança sempre é enfatizada por estabelecimentos comerciais e consumidores, porém é normalmente considerada como um Revista de Administração e Inovação, São Paulo, v. 10, n.3, p.89-114, jul./set. 2013. 
fator mais bem descrito como risco percebido pois apesar de que a maioria dos pagamentos realizados e' de pequeno valor caso a transação de alguma forma falha, o prejuízo financeiro não seria particularmente grande a impressão e' de insegurança pelos usuários. Estes usuários acreditam que a tomada de medidas de segurança adequadas minimizaria essa impressão e assim afetaria positivamente o risco percebido. Dahlberg et al. (2003) descreveram diversos tipos de riscos de segurança identificados em entrevistas, como o uso não autorizado ou erros nas transações. O primeiro ponto trás a preocupação de que terceiros consigam pagar com o aparelho eventualmente perdido ou roubado, enquanto o segundo ponto tem a ver com valores incorretos ou duplicados debitados de suas contas. Karnouskos (2004) destaca que os telefones móveis podem oferecer o estado da arte em segurança, e que soluções como I, biometria e assinaturas móveis digitais podem ser integradas facilmente nas arquiteturas de pagamentos móveis. Pousttchi (2003) conclui que a segurança não abrange somente integridade, autorização, autenticação, confidencialidade e não-repudiação de transações, mas também o problema de segurança subjetiva, segundo a perspectiva dos clientes.

Utilidade: Esta característica foi determinada desde a proposição inicial do modelo TAM, como o grau em que o usuário acredita que o sistema está melhorando a sua performance em um trabalho. Segundo Zmijewska et al. (2004b), no caso de pagamentos móveis, o trabalho seria entendido como algo trivial, composto por tarefas do dia a dia. O autor destaca que é muito importante que o sistema de pagamentos móveis ofereça para os usuários benefícios, incluindo a rapidez de transações. O sistema melhora a efetividade e eficiência da vida do usuário pela oferta de diversas aplicações, como compras, transferência entre pessoas, top ups, etc. Chen e Adams (2005) complementam que os usuários de telefones móveis podem se beneficiar da utilização de telefones com carteiras eletrônicas. Finalmente, os pagamentos móveis, segundo Mallat (2006), oferecem aos consumidores possibilidades ubíquas de compras. Os usuários podem pagar pelos serviços de meios de transportes ou estacionamentos, de forma remota, sem precisar ir a uma ATM, máquina de tickets ou parquímetros. Em conclusão, Dewan e Chen (2005) destacam que o consumidor terá mais propensão a adotar a tecnologia se ele perceber que o pagamento móvel é útil para seu processo de compra.

O Quadro 1 a seguir contem um resumo das características competitivas que impactam a decisão do usuário em adotar esta inovação. 


\begin{tabular}{|c|c|c|c|}
\hline & $\begin{array}{c}\text { Característica Competitiva que } \\
\text { impactam a adoção pelos } \\
\text { usuários }\end{array}$ & Descrição & Autores \\
\hline $\mathbf{A}$ & Custos & $\begin{array}{l}\text { Custos diretos da Transação } \\
\text { Custos Fixos de uso } \\
\text { Custos com novos equipamentos }\end{array}$ & $\begin{array}{l}\text { Karnouskos (2004), Mallat } \\
\text { (2006) e Pousttchi (2003) }\end{array}$ \\
\hline B & Compatibilidade & $\begin{array}{l}\text { Compatibilidade com os hábitos atuais do } \\
\text { usuário }\end{array}$ & $\begin{array}{l}\text { Chen e Adams (2005) e } \\
\text { Mallat (2006) }\end{array}$ \\
\hline $\mathbf{C}$ & Confiança & $\begin{array}{c}\text { Confiança no provedor para realizar o } \\
\text { cadastro no serviço } \\
\text { Reputação do Provedor } \\
\text { Confirmação do Pagamento e possibilidade } \\
\text { de Cancelamento }\end{array}$ & $\begin{array}{l}\text { Dahlberg et al. (2003), } \\
\text { Karnouskos (2004) e } \\
\text { Zmijewska et al. (2004b) }\end{array}$ \\
\hline D & Conveniência & $\begin{array}{c}\text { Conveniência para utilização } \\
\text { Locais para utilização } \\
\text { Redução da necessidade de dinheiro vivo }\end{array}$ & $\begin{array}{l}\text { Dewan e Chen (2005), } \\
\text { Karnouskos (2004), Mallat } \\
\text { (2006) e Pousttchi (2003) }\end{array}$ \\
\hline $\mathbf{E}$ & $\begin{array}{c}\text { Externalidades de Rede (Massa } \\
\text { Crítica) }\end{array}$ & $\begin{array}{c}\text { Valor para o usuário cresce quando existem } \\
\text { mais participantes } \\
\text { Criação de Massa crítica } \\
\text { Rede de estabelecimentos para uso } \\
\end{array}$ & Mallat (2006) \\
\hline $\mathbf{F}$ & Facilidade de uso & $\begin{array}{c}\text { Simplicidade da Solução } \\
\text { Interface Amigável } \\
\text { Pouca necessidade de aprendizagem para } \\
\text { uso } \\
\text { Capacidade de personalizar a solução }\end{array}$ & $\begin{array}{c}\text { Chen e Adams (2005), } \\
\text { Karnouskos (2004), Mallat } \\
\text { (2006) e Zmijewska et al. } \\
\text { (2004b) }\end{array}$ \\
\hline $\mathbf{G}$ & Mobilidade & $\begin{array}{c}\text { Possibilidade de utilização em qualquer } \\
\text { lugar } \\
\text { Cobertura de rede, roaming com outras } \\
\text { operadoras } \\
\text { Duração de bateria do device }\end{array}$ & $\begin{array}{l}\text { Dahlberg et al. (2003), } \\
\text { Mallat (2006) e Zmijewska } \\
\text { et al. (2004b) }\end{array}$ \\
\hline $\mathbf{H}$ & Observação de utilização & $\begin{array}{c}\text { Não usuários observam utilização de } \\
\text { usuários e se interessam }\end{array}$ & Chen e Adams (2005) \\
\hline $\mathbf{I}$ & Privacidade & $\begin{array}{l}\text { Anonimidade, } \\
\text { Confidencialidade } \\
\text { Integridade de dados } \\
\text { Autenticação }\end{array}$ & $\begin{array}{c}\text { Dahlberg et al. (2003), } \\
\text { Dewan e Chen (2005), } \\
\text { Karnouskos (2004), Mallat } \\
\text { (2006) e Zmijewska et al. } \\
\text { (2004b) }\end{array}$ \\
\hline $\mathbf{J}$ & Qualidade do Sistema & $\begin{array}{c}\text { Qualidade da rede de telefonia sem fio } \\
\text { Qualidade sistêmica da aplicação de } \\
\text { pagamento }\end{array}$ & Lee et al. (2004) \\
\hline $\mathbf{K}$ & Rapidez da Transação & $\begin{array}{c}\text { Diminuição do tempo da transação } \\
\text { Automatização da transação }\end{array}$ & $\begin{array}{l}\text { Dewan e Chen (2005), e } \\
\text { Zmijewska et al. (2004a) }\end{array}$ \\
\hline $\mathbf{L}$ & Segurança & $\begin{array}{c}\text { Percepção de segurança/risco na utilização } \\
\text { do serviço } \\
\text { Tecnologias (PKI, Biometria, Assinatura } \\
\text { digital) disponíveis } \\
\end{array}$ & $\begin{array}{c}\text { Dahlberg et al. (2003), } \\
\text { Karnouskos (2004), } \\
\text { Heijden (2002) e Pousttchi } \\
\text { (2003) } \\
\end{array}$ \\
\hline $\mathbf{M}$ & Utilidade & $\begin{array}{c}\text { Melhorar a performance do usuário para } \\
\text { funções: } \\
\text { compras, transferências entre pessoas, top } \\
\text { ups, etc. }\end{array}$ & $\begin{array}{l}\text { Chen e Adams (2005), } \\
\text { Dewan e Chen (2005), } \\
\text { Mallat (2006) e Zmijewska } \\
\text { et al. (2004b) }\end{array}$ \\
\hline
\end{tabular}

Fonte: Elaborado pelos autores

\section{$2.4 \quad$ Contexto ambiental}

Revista de Administração e Inovação, São Paulo, v. 10, n.3, p.89-114, jul./set. 2013. 


\subsubsection{Pagamentos móveis}

Pagamentos móveis são definidos como o uso de um equipamento móvel para realização de uma transação de pagamento, no qual o dinheiro ou os fundos são transferidos do pagador ao recebedor diretamente ou via um intermediário, segundo Mallat (2006). Sendo assim, complementam Dahlberg et al. (2007), pagamentos móveis são pagamentos por bens, serviços ou contas, com a utilização de um equipamento móvel. Karnouskos (2004) inclui ainda que qualquer pagamento em que um equipamento móvel foi utilizado para iniciar, ativar ou ainda confirmar um pagamento, pode ser considerado um pagamento móvel.

Zmijewska et al. (2004b) chamam a atenção para o fato de que o comércio eletrônico já foi um meio revolucionário que possibilita aos consumidores comprarem sem precisar sair de casa, a qualquer horário do dia ou da noite. Este tipo de compra normalmente era considerado como "em qualquer tempo, em qualquer lugar". Porém, este "qualquer lugar" sempre dependeria de acessar um computador em casa ou no trabalho. Os pagamentos móveis superaram esta limitação, sendo que agora os consumidores podem efetivamente comprar onde estiverem, com o seu telefone celular.

Dahlberg et al. (2007) destacam que os equipamentos móveis podem ser usados em uma variedade de tipos de pagamento como, por exemplo, pagamento por conteúdos digitais (ring tones, logos, notícias, música ou jogos), tickets, pagamento de estacionamento, tarifas de transporte, ou ainda para acessar serviços eletrônicos de pagamento para quitar contas ou faturas. Finalmente, são possíveis pagamentos de bens físicos, em terminais de captura de transações.

Dewan e Chen (2005) destacam que os equipamentos móveis podem ser telefones celulares, smart-phones, e ainda equipamentos com rádio freqüência ou a tecnologia de aproximação, chamada de "Near Field Communication" (NFC). Karnouskos (2004) complementa que ao contrário da percepção popular, pagamentos móveis não estão restritos somente a telefones celulares, mas também aos tablets PC ou PDAs.

Esta definição incluiria as transações de pagamentos móveis realizadas por sistemas de Mobile Banking, porém Mallat (2006) descreve uma diferenciação entre os dois tipos de serviços, sendo que o último é oferecido para os consumidores que tem relacionamento com uma instituição bancária, enquanto o primeiro é oferecido como uma nova forma de 
pagamento para o mercado varejista. Em conclusão, o negócio de pagamentos móveis é caracterizado por uma grande quantidade de fornecedores, como bancos e operadoras de telefonia, dois tipos diferentes de possíveis adotantes, consumidores e estabelecimentos comerciais, e finalmente a necessidade de uma padronização e compatibilização com os diferentes sistemas de pagamentos.

Todos estes fatores aumentam a complexidade do ambiente de adoção e uso de pagamentos móveis.

\subsubsection{O setor de meios de pagamento}

Os cartões de crédito e débito são produtos disponibilizados por instituições financeiras para serem utilizados por seus portadores na aquisição de bens e serviços, como forma de pagamento em estabelecimentos comerciais afiliados por empresas credenciadoras em processos definidos e regulados por uma bandeira.

Os participantes diretos do mercado de cartões são as bandeiras proprietárias dos esquemas, os portadores (compradores), os estabelecimentos (vendedores), os emissores e os credenciadores. Banco Central do Brasil [BACEN](2009).

O nome bandeira é ligado à "marca" que estará gravada nos cartões, sendo a proprietária dos seus direitos que definem os deveres de sua utilização. A bandeira também é responsável pela determinação das regras e padrões para operação, organizando a participação dos emissores e credenciadores na plataforma. Entre as principais atividades das empresas responsáveis pelas bandeiras, estão o desenvolvimento de novos produtos e serviços, criação de programas de marketing, publicidade e promoções. Operacionalmente, garantem o intercâmbio das informações decorrentes das transações feitas com cartões que possuam suas marcas interligando as credenciadoras aos emissores.

As principais bandeiras no mercado brasileiro são Visa, MasterCard, American Express, Hipercard e Diners, além de diversas outras de menor porte.

Os emissores são as instituições financeiras que vendem e emitem o cartão para seus clientes portadores, estampando seu nome no cartão, além da "marca" da bandeira de uso. $\mathrm{O}$ emissor também é responsável pelo relacionamento financeiro com seus clientes, garantindo para o sistema como um todo, o volume financeiro utilizado pelos portadores, sendo responsáveis pelo funding (operação de crédito) dos limites de crédito estabelecidos, bem como pelo risco de inadimplência a ele associado. Adicionalmente, os emissores têm como 
atividades a definição de políticas de risco, vendas e de programas de recompensas, a operação do atendimento aos clientes, faturamento e cobrança, além da execução de campanhas de marketing para fomentar o uso de seus produtos.

Os principais bancos brasileiros são emissores de cartões de crédito. Lojas e varejistas podem emitir cartões com marcas próprias, chamadas de cartões private label.

Credenciadores são responsáveis pela afiliação dos estabelecimentos para aceitação de uma certa bandeira. Eles são responsáveis pelas funções de análise de risco, credenciamento, relacionamento com os estabelecimentos e operação geral da captura de transações. Executam a intermediação dos fluxos financeiros entre emissores de cartões e estabelecimentos que aceitam o produto, capturando os dados transacionais para envio aos emissores, que finalmente concedem a autorização para que a venda ou pagamento de um serviço seja concluído. Finalmente, os credenciadores se responsabilizam pelos pagamentos aos lojistas referentes às compras feitas com cartões.

No Brasil, os principais credenciadores são Cielo e Redecard, porém existe um interesse muito grande de novos participantes entrarem neste mercado.

Além desses, existem outros agentes que atuam de forma direta ou indireta no setor, fazendo parte da rede de valor, como o provedor de terminal POS, o provedor de comunicação local, o fornecedor de cartões, os processadores, o prestador de serviço de rede (switch) e o prestador de serviços de liquidação, entre outros (BACEN, 2009).

\subsubsection{Os novos entrantes}

As operadoras de telefonia celular tem uma base de clientes muito grande e controlam os chips de telefonia instalados nos aparelhos. Sendo assim, estas operadoras podem influenciar e ter um impacto estratégico grande nos modelos de pagamentos móveis. Entretanto, conforme destacado por Karnouskos (2004), as operadoras não tem condições de gerenciar na totalidade os sistemas de pagamentos móveis, pela sua limitada experiência com meios de pagamentos, ou ainda pelos riscos associados com esta operação.

No Brasil, destacam-se as operadoras nacionais de telefonia celular Vivo, Claro e Oi, sendo que todas tem uma ou mais iniciativas neste novo mercado de serviços para seus consumidores.

Os fabricantes de equipamentos também têm um papel importante, pois eles controlam 
a tecnologia e capacidades do aparelho que o usuário receberá, afetando de forma importante a implementação e o desenvolvimento dos serviços de pagamentos móveis. Karnouskos (2004) chama a atenção para a necessidade dos fornecedores de equipamentos cooperarem entre si e com outros participantes do setor, para o desenvolvimento de uma abordagem comum das capacidades do aparelho móvel.

Finalmente, existem os fornecedores de softwares e serviços, que desenvolvem os meios de implantação de pagamentos móveis pela produção de sistemas padronizados e compatíveis que conectarão as diferentes partes do processo de pagamentos móveis. Ainda como fornecedores de serviços, oferecem as soluções para o mercado, adaptando-as para as necessidades dos usuários.

Karnouskos (2004) conclui que a cooperação entre todos os participantes do modelo de pagamentos móveis é chave para o desenvolvimento de soluções abertas e globais ao contrário de soluções de sistema fechado e de escopo limitado.

\section{PROCEDIMENTOS METODOLÓGICOS}

O problema delimitado para este estudo - qual a visão atual dos executivos relacionados ao mercado brasileiro de pagamentos móveis sobre os elementos de adoção pelo consumidor? - foi avaliado pela classificação das variáveis identificadas na revisão de literatura, segundo o Quadro 1.

\section{1}

\section{Natureza do estudo e plano amostral}

O estudo, de natureza qualitativa e de caráter exploratório, foi realizado com executivos de empresas participantes do mercado de meio de pagamentos brasileiro (emissores, bandeiras, operadoras de telefonia celular, fornecedores de software e soluções, fornecedores de equipamentos) que atuam no desenvolvimento ou gerenciamento de soluções de pagamentos móveis. Foram contatados dez executivos com o objetivo de se obter as informações sobre as características de adoção de soluções de pagamentos móveis. 
Foram realizadas entrevistas estruturadas com dirigentes de diversas empresas envolvidas no processo de desenvolvimento de soluções de pagamentos móveis no Brasil. As entrevistas tiveram como o objetivo coletar a percepção dos executivos sobre as características de adoção da inovação.

Durante a entrevista descreveu-se a metodologia de coleta de dados e explicou-se o objetivo de cada pergunta, e preencheu-se uma tabela de respostas de classificações.

A primeira pergunta realizada pretendia definir a importância de cada uma das características que impactam a adoção pelos usuários, segundo a percepção do executivo. Foi utilizada uma escala de "1" (ganhadores/muito importante) até "9 (pouco relevantes), com a escala "5" sendo a mediana (qualificadores).

P1: Qual a sua percepção da importância para os usuários de pagamentos móveis das características competitivas listadas?

Em seguida foram realizadas quatro perguntas para avaliar quatro soluções brasileiras que estão em processo de implantação, para identificação do desempenho de cada uma das características em relação a soluções competidoras.

P2 a P5: Na sua percepção, qual nota daria para o desempenho da solução (l a 4 ) para cada característica competitiva em relação a seus competidores?

Finalmente, foi realizada uma pergunta para avaliar o desempenho de cada uma das características em relação às soluções tradicionais de meios de pagamento.

P6: Na sua percepção, qual nota daria para o desempenho médio das soluções de pagamentos móveis para cada característica competitiva em relação aos meios de pagamento tradicionais?

A matriz de importância-desempenho oferece um método simples e muito útil para a consideração simultânea das dimensões de importância e performance para avaliar ou definir uma estratégia. Esta técnica tem sido utilizada com sucesso em uma série de estudos para definir prioridades e guiar as decisões de alocação de recursos.

Martilla e James (1977) apresentaram o modelo para auxiliar o processo de pesquisa de mercado com o objetivo de apoiar a compreensão da satisfação dos clientes em função das 
expectativas sobre as características mais importantes dos produtos, em conjunto com a avaliação do seu desempenho.

Slack (1994) propõe uma nova versão, com utilização de uma escala de 9 níveis, evoluindo a visão " $2 \times 2$ ” original deste instrumento.

Nesta nova versão, quatro zonas para análise são destacadas, "Adequada" (fatores de competição satisfatórios), "Melhorias" (fatores que necessitam serem aprimorados, mas não de maneira urgente), “Ações urgentes” (fatores mais críticos para análise) "Excesso?” (O ponto de interrogação é importante, pois deve ser avaliado se efetivamente utilizou-se muitos recursos para esta performance).

\section{ANÁLISE E INTERPRETAÇÃO DOS RESULTADOS}

Conforme o levantamento de informações pelo método de coleta utilizado neste trabalho, foi possível fazer alguns levantamentos e ponderações sobre as informações obtidas. Seguem, análises e interpretações dos dados coletados.

\subsection{Perfil dos respondentes}

No Quadro 2, segue a descrição dos perfis dos executivos da indústria de meios de pagamentos móveis que foram entrevistados.

Quadro 2. Perfil dos respondentes

\begin{tabular}{|c|c|}
\hline Natureza da Empresa & Posição da pessoa entrevistada \\
\hline Bandeira & Diretor de projetos \\
\hline Bandeira & VP produtos \\
\hline Emissor & Diretor de produtos \\
\hline Emissor & Gerente de projetos \\
\hline Fabricante de Aparelhos & Head desenvolvimento de negócios America Latina \\
\hline Fornecedora de equipamentos & Diretor tecnologia \\
\hline Fornecedora de Software & Diretor de serviços móveis \\
\hline Fornecedora de solução de pagamentos móveis & Diretor de tecnologia \\
\hline Fornecedora de solução de pagamentos móveis & Gerente Geral Brasil \\
\hline Operadora de telefonia móvel & Gerente Senior - Portfolio de pagamentos móveis \\
\hline
\end{tabular}

Fonte: Elaborado pelos autores

Revista de Administração e Inovação, São Paulo, v. 10, n.3, p.89-114, jul./set. 2013. 


\subsection{Resultados obtidos}

Para este estudo, avaliou-se a performance de quatro soluções em processo de implantação no Brasil, assim como a performance geral dos produtos de pagamentos móveis em relação aos meios de pagamento tradicionais.

A partir da avaliação das matrizes das Figuras 1,2,3 e 4, podem ser identificadas as características competitivas das soluções que estão sendo implantadas que merecem uma análise mais profunda, de forma mais crítica ou ainda o processo de melhorias que podem ser executadas nos processos de evolução destas soluções.

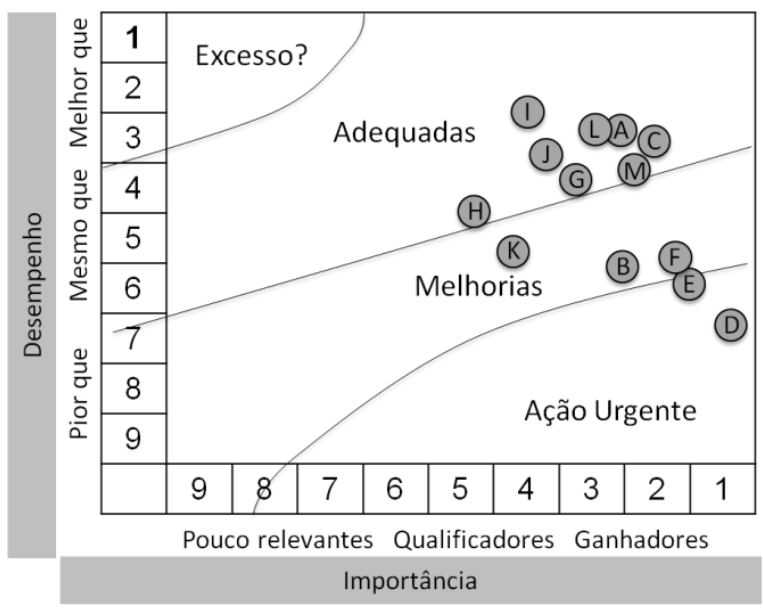

Figura 1: Matriz importância/desempenho Solução 1

Fonte: elaborado pelos autores

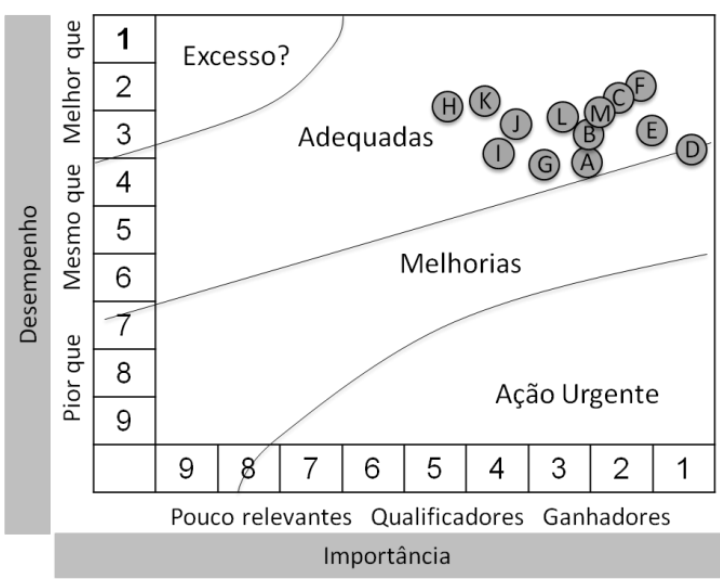

Figura 3: Matriz importância/desempenho

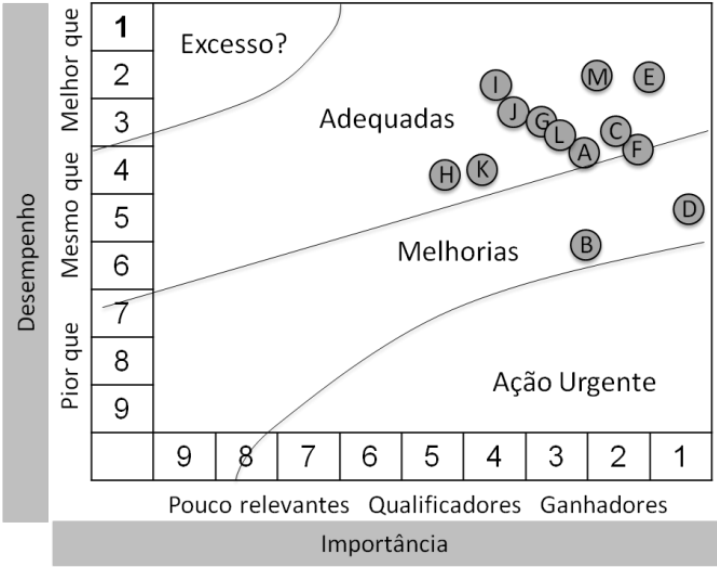

Figura 2: Matriz importância/desempenho Solução 2

Fonte: elaborado pelos autores

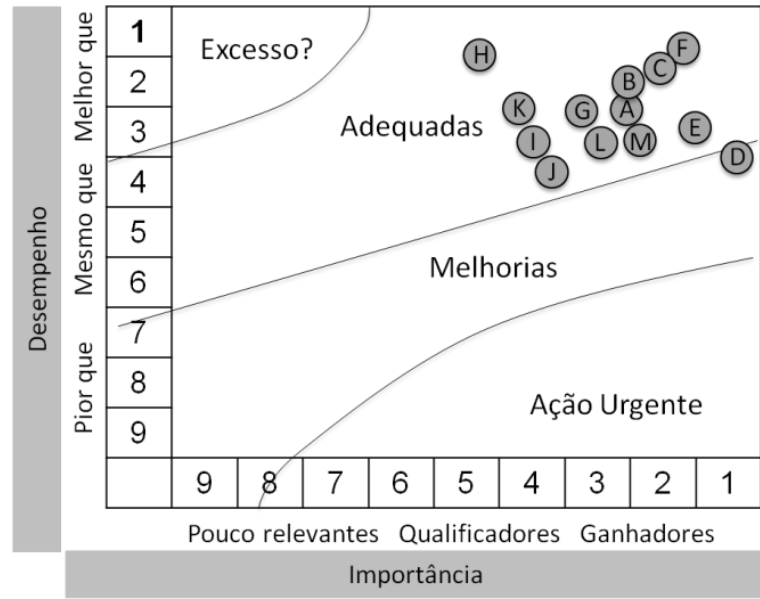

Figura 4: Matriz importância/desempenho

Revista de Administração e Inovação, São Paulo, v. 10, n.3, p.89-114, jul./set. 2013. 
Solução 3

Fonte: Elaborado pelos autores
Solução 4

Fonte: Elaborado pelos autores

Segundo a visão dos executivos pesquisados, a única solução em que foram identificadas características que mereceriam ações urgentes foi a "Solução 1", na qual os aspectos de conveniência (D) e externalidades de rede/massa crítica (E) mostraram-se como os principais pontos que deveriam preocupar os seus desenvolvedores, pontos estes já destacados por Dahlberg e Öörni (2006), Dewan e Chen (2005), Mallat (2006) e Pousttchi (2003).

Tanto na "Solução 1", quanto na "Solução 2", foram vistos que os aspectos de compatibilidade (B) e facilidade de uso (F) podem tornar-se desafios para melhorias no médio prazo. Especificamente na "Solução 1", aparece a rapidez da transação (K) como um ponto de melhoria. Na "Solução 2", identifica-se o item de conveniência (D), já crítico na solução anterior, como um ponto de melhoria.

Avaliando-se as "Soluções 3 e 4", percebe-se uma posição em melhores condições, com a percepção dos executivos indicando que quase a totalidade das características podem ser classificadas de forma adequada. A única característica em que foi identificada alguma necessidade de melhoria (muito mais pela importância dada do que pelo desempenho) foram novamente os aspectos de conveniência (D).

Analisando as quatro soluções, percebe-se que as características de Custos (A), Confiança (C), Mobilidade (G), Observação de Utilização (H), Privacidade (I), Qualidade do sistema (J), Segurança (L) e Utilidade (M) aparecem categorizadas como adequadas, demonstrando fatores de competição dentro do esperado pelos executivos entrevistados.

Nenhuma característica apareceu como em excesso, demonstrando que os executivos do mercado brasileiro, ao avaliarem as soluções disponíveis no mercado brasileiro atualmente, não identificaram características com um desempenho muito melhor que seus concorrentes ou aquelas pouco relevantes para os consumidores.

Revista de Administração e Inovação, São Paulo, v. 10, n.3, p.89-114, jul./set. 2013. 


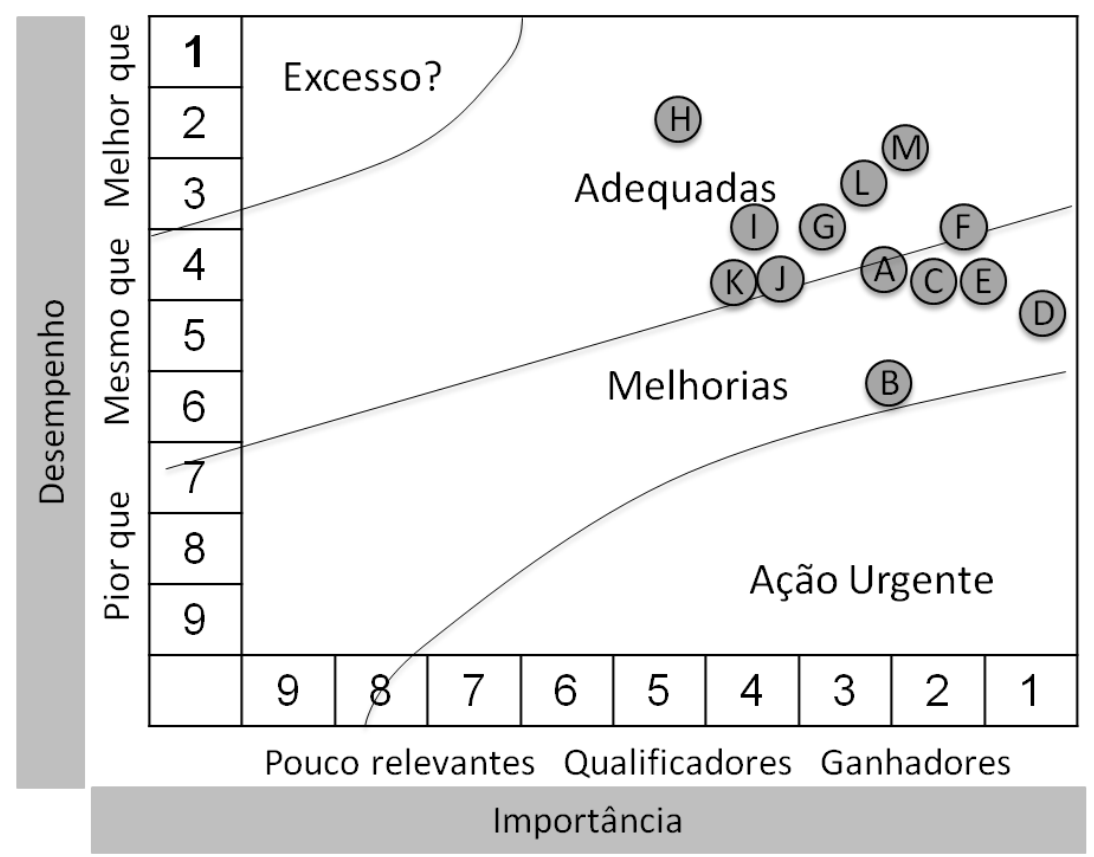

Figura 5. Matriz importância/desempenho - Comparação com meios de pagamento tradicionais Fonte: Elaborado pelos autores

A matriz de importância/performance acima permite a comparação das características das soluções de pagamento móveis em relação aos meios de pagamento tradicionais. Dahlberg, Mallat, Ondrus, Zmijewska (2007) propuseram que se estude as características dos serviços tradicionais de pagamentos que poderiam interferir na proliferação dos serviços de pagamentos móveis.

Avaliando-se os pontos considerados como ganhadores nos pagamentos móveis e que tenham um desempenho similar aos meios de pagamentos tradicionais, identificam-se as características que deveriam merecer maior atenção dos desenvolvedores de soluções. Novamente, as características de compatibilidade (B), conveniência (D), externalidades de rede/massa critica $(\mathrm{E})$ e facilidade de uso $(\mathrm{F})$ demonstraram estar em uma área de melhorias, da mesma maneira que a avaliação das soluções anteriores analisadas. Adicionalmente, encontra-se os itens de confiança (C) e de custos (A) como pontos importantes de atuação, quando se compara o pagamento móvel com os meios de pagamento tradicionais. Estes itens corroboram conclusões encontradas em estudo anterior de Dahlberg e Öörni (2006), realizado na Finlândia.

5CONSIDERAÇÕES FINAIS, LIMITAÇÕES DO ESTUDO E SUGESTÕES DE ESTUDOS FUTUROS

Revista de Administração e Inovação, São Paulo, v. 10, n.3, p.89-114, jul./set. 2013. 
Este estudo analisou as características de adoção das soluções de pagamento móveis e identificou que existem algumas que devem ser avaliadas com mais atenção pelos desenvolvedores destas soluções. Discutiu-se também, a emergência em que melhorias devem ou não ser feitas, segundo a percepção de executivos de empresas que atuam neste setor. A técnica utilizada foi a matriz importância/desempenho e os resultados demonstraram que os fatores ligados à característica de conveniência para o usuário de pagamentos móveis parece ser o item que merece mais atenção por parte dos desenvolvedores destes tipos de soluções. Conveniência pode ser caracterizada como a própria utilização, a existência de locais para consecução do meio de pagamento móvel, assim como a vantagem de redução da necessidade de dinheiro vivo. Outras características, que se destacaram como pontos de melhoria foram: a compatibilidade com os hábitos atuais do usuário, a facilidade de uso, representada pela simplicidade da solução, interface amigável, pouca necessidade de aprendizagem para uso e capacidade de personalizar a solução.

A literatura revisada destaca estudos que identificaram as principais características que impactariam a adoção deste tipo de inovação. Este estudo demonstrou uma metodologia de avaliação de tais características dentro de um contexto local e baseado em comparações com outras soluções e com os meios tradicionais de pagamento.

A avaliação final deste trabalho destaca também os fatores das soluções que parecem estar adequados para as ofertas atuais, como: mobilidade, observação de utilização, aspectos de privacidade e segurança, qualidade dos sistemas e utilidade.

Este estudo não captou a percepção dos usuários finais, tendo utilizado como base a literatura disponível. Este situação pode ter trazido um viés da visão dos executivos entrevistados.

Como proposta de estudo futuros, pode-se efetivamente aprofundar em cada uma das soluções, destacando as características de cada uma em relação aos meios tradicionais de pagamento. Outra alternativa seria a ampliação da análise com outras empresas da cadeia de valor.

Entende-se que este estudo tem uma importante implicação para os desenvolvedores de soluções uma vez que, identificando-se os fatores que facilitariam a adoção do usuário final que estejam em um nível de oferta não adequado ao que opções competidoras oferecem, os gestores podem realizar suas escolhas para aplicação de recursos na melhora desta oferta 
final ao consumidor. O estudo demonstra que se podem encontrar respostas para estas questões.

\section{REFERÊNCIAS}

Afuah, Allan.(1998). Innovation Management: Strategies, Implementation, and Profits (2a ed.). New York, USA: Oxford University. Press.

BACEN. Banco Central do Brasil. (2009). Relatório sobre a Indústria de Cartões de Pagamentos, 1a edição, maio/2010. Recuperado em 02 de setembro de 2011, de https://www.bcb.gov.br/htms/spb/Relatorio_Cartoes.pdf

Chen, J. \& Adams, C. (2005, December). User acceptance of mobile payments: a theoretical model for mobile payments. Proceedings of the Fifth International Conference on Electronic Business (ICEB), Hong Kong, 5-9.

Dahlberg, T., Mallat, N., Ondrus, J., \& Zmijewska, A. (2007). Past, present and future of mobile payments research: A literature review. Electronic Commerce Research and Applications, 7(2), 165-181.

Dahlberg, T., Mallat, N., \& Öörni, A. (2003). Trust enhanced technology acceptance modelconsumer acceptance of mobile payment solutions: Tentative evidence. Stockholm Mobility Roundtable, 22-23.

Dahlberg, T.\& Öörni, A. (2006, June). Understanding changes in consumer payment habits do mobile payments attract consumers? Presentation at Helsinki Mobility Roundtable, Helsinki, Finland, 1-2.

Davis, F. D. (1989). Perceived usefulness, perceived ease of use, and user acceptance of information technology. MIS quarterly, 319-340.

Davis, F., Bagozzi, R. \& Warshaw, P.(1986). User acceptance of computer technology: a comparison of two theoretical models. Management Science. 35 (8), 982-1002.

Dewan, S. G., \& Chen, L. D. (2005). Mobile payment adoption in the USA: a cross-industry, crossplatform solution. Journal of Information Privacy \& Security, 1(2), 4-28.

Frambach, R. T., \& Schillewaert, N. (2002). Organizational innovation adoption: A multilevel framework of determinants and opportunities for future research. Journal of Business Research, 55(2), 163-176.

Heijden, H. (2002, June). Factors affecting the successful introduction of mobile payment systems. 15th Bled Electronic Commerce Conference (Bled 2002), Bled, Slovenia. 
Karnouskos, S. (2004). Mobile payment: a journey through existing procedures and standardization initiatives. Communications Surveys \& Tutorials, IEEE, 6(4), 44-66.

Kleijnen, M., Wetzels, M., \& de Ruyter, K. (2004). Consumer acceptance of wireless finance. Journal of Financial Services Marketing, 8(3), 206-217.

Lee, C. P., Warkentin, M., \& Choi, H. (2004). The Role of Technological and Social Factors on the Adoption of Mobile Payment Technologies. AMCIS 2004 Proceedings, 333.

Mallat, N. (2006). Exploring consumer adoption of mobile payments-a qualitative study. The Journal of Strategic Information Systems, 16(4), 413-432.

Martilla, J. A., \& James, J. C. (1977). Importance-performance analysis. The journal of marketing, 77-79.

OCDE. Organisation of Economic Co- Operation and Development. (1994). The measurement of Scientific and Techonological Activities. Proposed Guidelines for Collecting and InterpretingTechnological Innovation Data - OSLO MANUAL. Recuperado em: 12 agosto de 2011, de http://oecd.com

Pousttchi, K. (2003). Conditions for acceptance and usage of mobile payment procedures. The Second International Conference on Mobile Business

Slack, N. (1994). The importance-performance matrix as a determinant of improvement priority. International Journal of Operations \& Production Management, 14(5), 59-75.

Tidd, J., Bessant, J.\& Pavitt, K. (2005). Managing Innovation - Integrating Technological, Market and Organizational Change, John Miley \& Sons, 3.ed..

Zmijewska, A., Lawrence, E., \& Steele, R. (2004). Towards a successful global payment system in mobile commerce. IADIS International E-Commerce, Lisbon, Portugal.

Zmijewska, A.; Lawrence, E.\& Steele, R. A. (2004b). Towards understanding of factors influencing user acceptance of mobile payment systems. Proceedings of the IADIS WWW/Internet, Madrid, Spain, October 6-9. 


\title{
COMPETITIVE CHARACTERISTICS THAT IMPACT MOBILE PAYMENT ADOPTION
}

\begin{abstract}
This paper aims to argue the competitive characteristics that would impact the adoption of mobile payments for the end user under the perception of this sector executives, in order to figure out a answer to the following question: what is the current vision from the executives related to the Brazilian mobile payment market regarding the consumer innovation adoption elements. This paper also intends to contextualize and describe the participants on this scheme. The exploratory paper was developed by means of structured interviews with ten executives representing the mobile payment market. The gotten data had been analyzed by the technical of importance performance matrix and the results indicate that convenience, compatibility with the current user habits and ease of use are the competitive characteristics of mobile payments solutions that should deserve more attention from the solutions developers, to enhance the consumer adoption level.
\end{abstract}

Keywords: Business; Inovation; Innovation Adoption; Mobile Payments; Importance Performance Matrix.

Data do recebimento do artigo: 12/12/2012

Data do aceite de publicação: 01/07/2013

Revista de Administração e Inovação, São Paulo, v. 10, n.3, p.89-114, jul./set. 2013. 